\section{PSICOLOGIA IBEROAMERICANA}

\section{Psicología lberoamericana}

ISSN: 1405-0943

psicología.iberoamericana@uia.mx

Universidad Iberoamericana, Ciudad de

México

México

Ramírez Landeta, Jorge José; Álvarez Guerra, Guillermo Jesús Características Demográficas Asociadas a los Estilos de Manejo de Conflicto en Gerentes Venezolanos

Psicología Iberoamericana, vol. 13, núm. 1, 2005, pp. 35-42

Universidad Iberoamericana, Ciudad de México

Distrito Federal, México

Disponible en: http://www.redalyc.org/articulo.oa?id=133926982006

Cómo citar el artículo

- Número completo

- Más información del artículo

- Página de la revista en redalyc.org

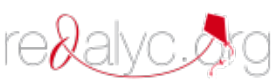

Sistema de Información Científica

Red de Revistas Científicas de América Latina, el Caribe, España y Portugal

Proyecto académico sin fines de lucro, desarrollado bajo la iniciativa de acceso abierto 


\title{
Características Demográficas Asociadas a los Estilos de Manejo de Conflicto en Gerentes Venezolanos
} Demographic Characteristics Associated to the Styles
of Handling Conflict in Venezuelan Managers

\author{
Jorge José Ramírez Landeta* y Guillermo Jesús Álvarez Guerra \\ Universidad Stmón Bolfvar, Venezuel.a
}

\section{Resumen}

El propósito de la presente investigación es determinar el estilo de manejo de conflictos predominante utilizado por gerentes del Área Metropolitana de Caracas y estudiar la influencia de algunas caracteristicas demográficas sobre los mismos. Se seleccionó una muestra de 558 profesionales universitarios. Se aplicó un cuestionario de caracteristicas demográficas y el inventario de estilo de manejos de conflicto. Los resultados obtenidos muestran que los gerentes tienden a comportarse predominantemente según el estilo Integrador. Con relación a las características demográficas estudiadas, se determinó que el género femenino tiende a comportarse de forma más integradora y comprometida que el género masculino; no se encontró influencia de la edad sobre los estilos de manejo de conflicto en los gerentes.

Descriptores: manejo de conflictos, comportamiento organizacional, métodos gerenciales, interacción interpersonal, equipos de trabajo

\begin{abstract}
The purpose of the present investigation was to determine the style of handling conflicts used by managers of the Metropolitan Area of Caracas and study the influence of some demographic characteristics on the handling of conflicts. A sample of 558 university professionals was selected. A questionnaire of demographic characteristic was applied as well as the inventory of style of conflict handling. The obtained results show that the managers behave predominantly according to the Integrative style. In relation to the studied demographic characteristics, the feminine gender behaves in a more integrative and committed way than the masculine gender, age was not an influence on the styles of conflict handling.
\end{abstract}

Descriptors: conflict management, organizational behavior, management methods, interpersonal interaction, work teams

\section{Introducción}

El papel gerencial supone una función de influencia sobre los miembros de la organización cuyo propósito es vincular la conducta humana de los trabajadores con el logro de los objetivos de la organización, a través de la interacción entre sus miembros y el manejo de conflictos potenciales derivados de la misma (Rahim, 2000).

La palabra conflicto proviene del latín conflictus, y significa choque, oposición. El término tiene una fuerte carga semántica que actúa como disparador de otros conceptos, ideas y sentimientos usualmente desagradables (Rahim, 1983).

El conflicto, de forma general, se entiende como inherente a la naturaleza humana y se manifiesta en todos los niveles de las relaciones: intrapersonales, interpersonales, grupales, organizacionales, nacionales e internacionales. El tema del conflicto es inherente al ser humano $\mathrm{y}$ ha influenciado el pensamiento desde tiempos inmemoriales. Éste ha recibido de los cientificos sociales diferentes grados de énfasis du-

\footnotetext{
- Dirigir correspondencia a: A partado postal 89000, Caracas, Venezuela. Correo electrónico: jjramirezgusb.ve
} 
rante varios periodos de la historia y ha sido objeto de interés para historiadores, novelistas, filósofos, juristas y teólogos, entre otros. El conflicto es omnipresente, por lo que no hay forma de vivir sin él (Hammond, 1999; Rahim \& Afza, 1993).

El conflicto interpersonal en las organizaciones es una respuesta natural de la interacción humana que comienza cuando un individuo percibe que sus metas, intereses, actitudes, valores o creencias son incongruentes o diferentes con aquellas de otros individuos. Cada persona en la organización tiene diversas formas de manejarse frente a los conflictos interpersonales y el estilo de manejo del conflicto resulta un aspecto de gran relevancia en las relaciones organizacionales (Rahim, 1983, 2000).

La concepción del conflicto en las organizaciones ha experimentado una evolución a lo largo de los siglos. En los inicios, los expertos de la administración cientifica, como Frederick W. Taylor, consideraban que todos los conflictos representaban una amenaza para la autoridad de la dirección y por consiguiente que habia que evitarlos o resolverlos lo más rápidamente posible (Kreitner \& Kinicki, 1996; Rahim \& Afza, 1993). Más adelante, los expertos en relaciones humanas reconocieron el carácter inevitable de los conflictos y aconsejaron a los directivos a aprender a convivir con ellos como parte de las organizaciones, pero con énfasis en resolverlos. Sin embargo, no fue sino hasta inicio de la década de los años sesenta cuando los investigadores en comportamiento organizacional manifestaron la novedosa perspectiva sobre cómo los conflictos podían tener resultados positivos o negativos, en función de su naturaleza y de su necesidad; se sustentó incluso la idea que las organizaciones podian ser víctimas de la falta de conflictos (Robbins, 1998; Kreitner \& Kinicki, 1996; Rahim \& Afza, 1993; Rahim, 2000).

Con la finalidad de aproximarse a los conflictos interpersonales propios del ambiente organizacional, Rahim (1983), aplicando una conceptualización similar a la de Blake \& Mounton (1964) y de Thomas (1976), define que los estilos de manejo de conflictos interpersonales se manifiestan en dos dimensiones básicas: concerniente a sí mismo y concerniente a los otros. La primera dimensión explica el grado (alto o bajo) en que una persona intenta satisfacer sus propias preocupaciones. La segunda dimensión explica el grado (alto o bajo) en que una persona quiere satisfacer las preocupaciones de los otros. Estas dimensiones reflejan las orientaciones que motivaban al individuo durante una situación de conflicto (Thomas, 1976; Van de Vliert \& Kabanoff, 1990).

Rahim (1983) definió el estilo de manejo de conflicto como un patrón de respuesta o modo característico de manejar una situación conflictiva en la organización. Postula cinco estilos de manejo de conflictos: integrativo, complaciente, dominante, evitativo y comprometido, como se muestra en la figura 1 .

\section{Figura 1. Modelo de dos dimensiones de los estilos interpersonales de manejo de conflictos (Rahim, 1983)}

Concerniente a sí mismo

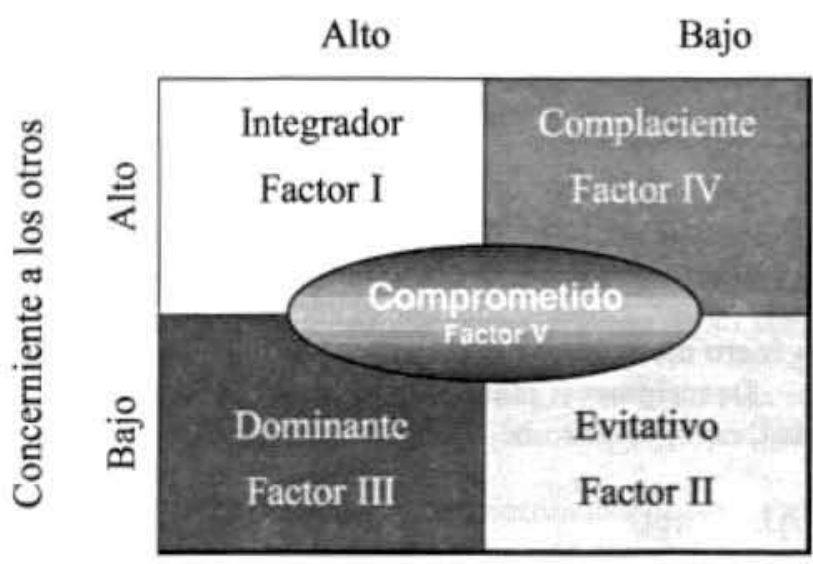

El estilo evitativo involucra baja preocupación por sí mismo así como por los demás; también se llama inactivo, reactivo, o el estilo ignorado. Está asociado con situaciones esquivas o de retirada, "no ve nada malo", "no oye nada malo" y "no habla acerca de situaciones malas", pospone el problema hasta un mejor tiempo o simplemente se retira de la situación amenazante. No satisface ni sus preocupaciones ni las de los demás. Este estilo se caracteriza a menudo por una actitud indiferente hacia los problemas o partes involucradas en el conflicto. Puede negarse a reconocer en público que hay un conflicto que debe ser abordado (Rahim, 1983, 2000).

El estilo integrador involucra la preocupación concerniente a sí mismo como a las otras partes, también se ha descrito como el de personas que solucionan problemas con una visión ganar-ganar. Involucra la colaboración activa entre las partes (es decir, franco intercambio de información y examina las diferencias) para alcanzar una solución que satisfaga las preocupaciones de las partes. Rahim (2000) sugirió que este estilo tiene dos elementos distintivos: la confronta- 
ción, la cual involucra una comunicación abierta y directa hasta llegar a una vía que permita solucionar el problema y la resolución de éste.

El estilo complaciente involucra baja preocupación por sí mismo y alta por las otras partes. También es llamado cómodo, porque no confronta, se rinde, complaciente o estilo perder-ganar. Es asociado con un perfil bajo en sus diferencias y enfatiza las comodidades para satisfacer a las otras partes. Hay un elemento de autosacrificio en este estilo que puede tomar forma de minusvalia, generosidad, caridad u obediencia a las órdenes de las otras personas. Una persona complaciente descuida sus propias preocupaciones para satisfacer las de las otras partes. Tal individuo tiende a absorber los conflictos de los otros; es decir, una persona cuya reacción a los actos de hostilidad percibida por parte de los otros tiende a ser positiva e incluso amigable (Rahim, 2000).

El estilo dominante involucra alta preocupación por sí mismo y baja preocupación por los demás. Es llamado también competidor, controlador, contendor o cero aporte. Ha sido identificado con estilo ganarperder y con conductas para ganar la posición numero uno. Una persona dominante sale para cumplir sus objetivos y como resultado a menudo ignora las necesidades y expectativas de la otra parte. Dominante puede significar anteponer sus derechos y/o defender una posición que las personas creen correcta. A veces una persona dominante quiere ganar cueste lo que cueste (Rahim, 2000).

El estilo comprometido involucra la preocupación moderada por sí mismo, así como por los demás. También se llama el estilo de motivación mixta en la teoría. Involucra dar y recibir o compartir, en situaciones en que ambas partes llegan a decisiones mutuamente aceptables. La persona comprometida típicamente logra el fraccionamiento de la diferencia, o buscar el punto medio de las posiciones, a fin de asegurar cumplir con los objetivos (Rahim, 2000).

En el ámbito venezolano, las organizaciones y los gerentes no escapan de las potenciales influencias de los conflictos y sus repercusiones (positivas o negativas). Sin embargo, en la actualidad existe escasa información proveniente de investigaciones cientificas sobre el comportamiento de los gerentes y los estilos de manejo de conflictos interpersonales utilizados en el ambiente organizacional.

En este sentido, los objetivos del presente estudio fueron los siguientes: primero, determinar el estilo de manejo de conflictos predominante utilizado por gerentes del Área Metropolitana de Caracas. El segun- do, identificar la influencia de algunas caracteristicas demográficas sobre el estilo predominante de manejo de conflictos.

Dos razones justificaron la investigación: desde el punto de vista teórico, conocer sobre las posibles relaciones entre variables asociadas con los estilos de manejo de conflicto en gerentes venezolanos constituye un recurso cientifico relevante para las disciplinas que buscan maximizar el potencial humano en los ambientes organizacionales. Al respecto, la presente investigación pretendió contribuir en generar datos e información sobre las relaciones entre algunas variables demográficas asociadas a los estilos de manejo de conflicto. Desde el punto de vista de la gerencia venezolana, disponer de información cientifica sobre las tendencias de uso de los estilos de manejo de conflicto constituye un valioso recurso que podría redundar en una mejor comprensión de la ejecución y desenvolvimiento, así como en elaboración del diseño de programas de adiestramiento y actualización académica que redunden en su beneficio. En este sentido, se espera realizar una contribución en la comprensión sobre cómo los gerentes venezolanos manejan los conflictos interpersonales en la organización.

\section{Método}

Las variables independientes fueron: a) género del gerente $\mathrm{y} b$ ) edad del gerente. La variable dependiente fue el estilo de manejo de conflictos, entendida como un patrón de respuesta o modo característico de manejar una situación conflictiva en la organización (Rahim, 1983, 2000). La misma se mide por el resultado que el participante obtiene de la aplicación del Inventario de Conflictos Organizacionales de Rahim II, el cual evalúa los cinco estilos propuestos: $a$ ) estilo integrador, b) estilo complaciente, c) estilo dominante, d) estilo evitativo y $e$ ) estilo comprometido.

La muestra estuvo constituida por 558 trabajadores seleccionados de forma intencional, aleatoria, profesionales universitarios, pertenecientes a empresas públicas y privadas del Área Metropolitana de Caracas, matriculados como estudiantes de posgrado de las áreas de ciencias administrativas, empresariales, gerenciales (Universidad Central de Venezuela, Universidad Simón Bolívar, Universidad Católica Andrés Bello); de género masculino (257) y de género femenino (301), cuyas edades están comprendidas entre 23 y 60 años y desempeñan un papel en la organización como gerentes en formación, gerencia baja, gerencia media y gerencia alta. 
El instrumento utilizado fue el Inventario de Estilo de Manejo de Conflictos ROCI-II, de Rahim (1983) en su forma "A", el cual fue adaptado a la población venezolana por Ramírez y Álvarez (en prensa). La consistencia interna del instrumento, medida por el Alfa de Cronbach es reportada de .84 , así como que cada una de sus cinco subescalas varían entre .65 y .82 . Dichas subescalas representan cada uno de los cinco factores teóricos de estilos de manejo de conflicto.

En el procedimiento utilizado para la realización de la investigación, en primer lugar se contactaron tres centros de educación universitaria a nivel de posgrado, pertenecientes al Área Metropolitana de Caracas, en los cuales se dictan justamente posgrados en el área de Ciencias Administrativas y Gerenciales. Se solicitó permiso a sus autoridades, así como a cada uno de los participantes. Se procedió a la aplicación del instrumento presentado en una hoja tamaño carta, por una cara el cuestionario de datos demográficos y por la otra el inventario de manejo de conflictos. El instrumento fue aplicado de forma directa por los investigadores, en una sola oportunidad, siguiendo un conjunto estandarizado de instrucciones.

\section{Análisis y discusión de los resultados}

Se presenta en primer lugar la distribución de la población en cuanto a las características demográficas. Posteriormente se presentan los procedimientos utilizados para el logro de los objetivos propuestos.

La distribución de la muestra en cuanto a la variable género se presenta de la siguiente forma: 301 fueron de género femenino $(53.9 \%)$ y 257 de género masculino $(46.1 \%)$. En cuanto a la variable edad de los participantes, se conformaron dos categorías, menores o iguales a 30 años, con 296 participantes $(53.22 \%)$ y mayores de 31 años, conformada con 261 participantes $(46.78 \%)$. Se observa que para la categoría menor o igual a 30 años, la mayoría de los participantes son de género femenino (59.93\%), mientras que en la categoría mayor a 31 años, el género masculino resulta conformar el mayor grupo $(52.87 \%)$ (véase tabla 1).

Para determinar el estilo de manejo de conflictos predominante utilizado se realizó el análisis de la frecuencia de uso de los estilos de manejo de conflicto (tabla 2), y el análisis de las medias del nivel de uso (tabla 3 y figura 1). El estilo integrador, utilizado por $61,8 \%$ de la muestra (345 participantes) y el estilo comprometido utilizado por $28.9 \%$ de la muestra ( 171 participantes) (véase tabla 2 ).
Tabla 1. Género y edad de los participantes

\begin{tabular}{|l|c|c|c|c|c|c|}
\hline \multirow{2}{*}{ Edad } & \multicolumn{6}{|c|}{ Genero } \\
\cline { 2 - 7 } & \multicolumn{2}{|c|}{ Femenino } & \multicolumn{2}{c|}{ Masculino } & \multicolumn{2}{c|}{ Total } \\
\cline { 2 - 7 } & $N$ & $\%$ & $N$ & $\%$ & $N$ & $\%$ \\
\hline $\begin{array}{l}\text { Menor o igual } \\
\text { a 30 años }\end{array}$ & 178 & 59.93 & 119 & 40.07 & 297 & 100 \\
\hline Mayor a 31 años & 123 & 47.13 & 138 & 52.87 & 261 & 100 \\
\hline Total & 301 & & 257 & & 558 & \\
\hline
\end{tabular}

Tabla 2. Frecuencia de uso de los estilos de manejo de conflicto en gerentes del Área Metropolitana de Caracas

\begin{tabular}{|l|c|r|}
\hline & Frecuencia & $\%$ \\
\hline Integrador & 345 & 61.8 \\
\hline Comprometido & 161 & 28.9 \\
\hline Evitativo & 21 & 3.8 \\
\hline Complaciente & 16 & 2.9 \\
\hline Dominante & 15 & 2.7 \\
\hline Total & 558 & 100.0 \\
\hline
\end{tabular}

Se calcularon las medias del nivel de uso de los estilos de manejo de conflicto que se muestran en la tabla 3. El estilo integrador posee la media más alta: 4.309 y la desviación típica más baja: .476, de los cinco estilos evaluados. Asi mismo, se muestra la preferencia de los otros estilos, ordenados según la media, lo que permite determinar que el estilo de manejo de conflicto integrador posee la media más alta y resulta ser el estilo más frecuentemente utilizado. Dicho análisis es el procedimiento sugerido por Rahim (1983) y Sirivum (2001) para determinar el estilo de manejo de conflicto predominante.

En la figura 2 se muestra un diagrama de cajas que permite observar el comportamiento de los cinco estilos de manejo de conflicto. El estilo integrador presenta la mediana estadistica más alta de los cinco estilos de manejo de conflicto, $50 \%$ de los participantes (entre el cuartil 25 y 75 representado por la caja) obtienen los valores más altos de la escala utilizada (entre 4 y 5 puntos).

El estilo comprometido presenta la segunda mediana más alta; sin embargo, $50 \%$ de los participantes presentan valores entre 3.75 y 4.25 . Los estilos complaciente, evitativo y dominante, presentan medianas muy similares entre sí (cercanas a 3 ) pero muy inferiores a la mediana del estilo integrador que resultó ser el predominante. 
Tabla 3. Nivel de uso de los estilos de manejo de conflicto en gerentes venezolanos

\begin{tabular}{|l|c|c|c|c|c|}
\hline & Integrador & Comprometido & Complaciente & Evitativo & Dominante \\
\hline Uso & 1 er. & 2 do. & 3ero. & 4 to. & 5to. \\
\hline Media (M) & 4.309 & 4.003 & 3.026 & 3.018 & 3.014 \\
\hline Desviación típica (S) & .476 & .558 & .717 & .792 & .709 \\
\hline Minimo & 2 & 2 & 1 & 5 & 1 \\
\hline Máximo & 5 & 5 & 5 & .627 & 5 \\
\hline Varianza & .226 & .312 & .514 & 503 \\
\hline
\end{tabular}

Figura 2. Gráfico de caja de los estilos de manejo de conflictos

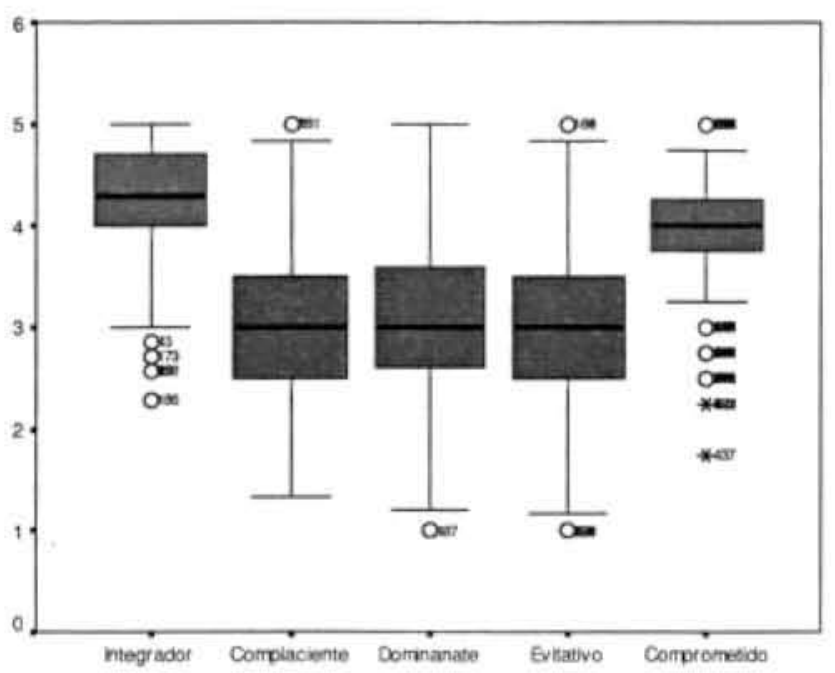

Los resultados de las pruebas de frecuencia de uso y las medias de los estilos de manejo de conflicto constituyen un cuerpo de evidencia que permite inferir que el estilo de manejo de conflicto predominante tiende más a integrador y comprometido que a complaciente, dominante y evitativo.

Para determinar la influencia de algunas características demográficas (género y edad) sobre el estilo de manejo de conflictos predominante se procedió a examinar la diferencia de medias según el género, para el estilo predominante, integrador, así como en el resto de los estilos, con una prueba $t$ para grupos independientes. Se utilizó un intervalo de confianza de $95 \%$ y un nivel de significación de 0.05 . Los resultados generales pueden verse en la tabla 4 .

Al observar los estilos de manejo de conflicto según el género, se aprecia que tanto para el género femenino como para el masculino la media más alta

Tabla 4. Estilos de manejo de conflictos según géneros

\begin{tabular}{|c|c|c|c|c|c|c|}
\hline \multirow{2}{*}{ Estilo } & \multirow{2}{*}{ Género } & \multirow{2}{*}{ Media } & \multirow{2}{*}{ Desviación tipica } & \multicolumn{3}{|c|}{ Prueba $T$} \\
\hline & & & & $T$ & $G L$ & Sig. \\
\hline \multirow{2}{*}{ Integrador } & Femenino & 4.371 & .478 & 3.354 & 556 & $.001^{*}$ \\
\hline & Masculino & 4.237 & .464 & & & \\
\hline \multirow{2}{*}{ Comprometido } & Femenino & 4.077 & .579 & 3.425 & 556 & $.001^{\bullet}$ \\
\hline & Masculino & 3.916 & .521 & & & \\
\hline \multirow{2}{*}{ Complaciente } & Femenino & 3.072 & .750 & 1.642 & 556 & .101 \\
\hline & Masculino & 2.972 & .675 & & & \\
\hline \multirow{2}{*}{ Evitativo } & Femenino & 3.060 & .827 & 1.343 & 556 & .952 \\
\hline & Masculino & 2.970 & .748 & & & \\
\hline \multirow{2}{*}{ Dominante } & Femenino & 3.012 & .728 & -0.060 & 556 & .180 \\
\hline & Masculino & 3.016 & .687 & & & \\
\hline
\end{tabular}

- Significativa $<0.001$ 
resulta ser la del estilo integrador (femenino $=4.371$ y masculino $=4.237)$ y la segunda media más alta resultó ser el estilo comprometido (femenino $=4.077$ y masculino $=3.916$ ). Sin embargo, el resto de las medias se distribuyen de forma diferente según el género.

Los resultados de la prueba $t$ señalan que para el estilo de manejo de conflicto integrador se encontraron diferencias significativas entre el género femenino y masculino y el uso del estilo $t(556)=3.35$, $\mathrm{p}<0.001 \mathrm{y}$ al análisis de ambas medias según el género, se observa que el femenino fue de 4.371 y el masculino fue de 4.237 , lo que permite afirmar que el género femenino tiende a comportarse de forma más integradora que el género masculino. Asimismo, al analizar los resultados de la prueba $t$, para el segundo estilo más frecuente, comprometido, se observa que existen diferencias significativas entre el género femenino $(M=4.077)$ y masculino $(M=3.916)$ y el uso de este estilo, $t(556)=3.43, \mathrm{p}<0.001$, a favor del género femenino. Sin embargo, las pruebas $t$ aplicadas a los estilos complaciente, evitativo y dominante para evaluar las diferencias según el género, señalan que no existe diferencias estadísticamente significativas para estos estilos de manejo de conflicto.

En cuanto a la relación: edad y estilo de manejo de conflictos, se realizó una prueba $t$ para grupos independientes para examinar la diferencia entre medias de la frecuencia de uso del estilo predominante, integrador, y la edad del gerente. Se utilizó un intervalo de confianza de $95 \%$ y un nivel de significación de 0.05 ; los resultados para el estilo integrador $t(556)=-.534$, $\mathrm{p}=0.595$, señalan que la diferencia encontrada no resulta significativamente estadística (véase tabla 5).

\section{Discusión}

Los gerentes del Área Metropolitana de Caracas tienden a comportarse preponderantemente de forma integradora para abordar los conflictos interpersonales que se presentan en las organizaciones. Como principales características del gerente integrador se tiene que suele considerar las preocupaciones concernientes a sí mismo, como a las otras partes involucradas a la hora de resolver los conflictos; se le describe como persona preactiva, orientada a la solución de problemas; resultan colaboradores y establecen relaciones "ganar-ganar" en las que todas las partes ganan y aportan en la solución. Asimismo, concentran su esfuerzo en alcanzar una solución que satisfaga a las partes involucradas mediante la búsqueda activa de colaboración, el examen de las diferencias existentes y el intercambio de información (Rahim, 2000).

El gerente integrador involucra la colaboración activa entre las partes a través de un franco intercambio de información y análisis de las diferencias para alcanzar una solución que satisfaga las preocupaciones de las partes. "La primera regla para obtener la integración es ser capaz de poner las diversas posiciones de forma clara en la mesa" (Rahim, 2000, p. 10); esto implica enfrentar el problema real, destapar

Tabla 5. Estilos de manejo de conflicto según la edad

\begin{tabular}{|l|c|c|c|c|c|c|}
\hline \multirow{2}{*}{} & \multirow{2}{*}{ Edad } & \multirow{2}{*}{ Media } & \multirow{2}{*}{ Desviación tipica } & \multicolumn{3}{|c|}{ Prueba t } \\
\cline { 5 - 7 } & & & & $t$ & GL & Sig. \\
\hline \multirow{2}{*}{ Integrador } & Menor o igual a 30 & 4.299 & .476 & -0.534 & 556 & .594 \\
& Mayor o igual a 31 & 4.321 & .477 & & & \\
\hline \multirow{2}{*}{ Comprometido } & Menor o igual a 30 & 4.043 & .550 & 1.799 & 556 & .073 \\
& Mayor o igual a 31 & 3.958 & .565 & & & \\
\hline \multirow{2}{*}{ Complaciente } & Menor o igual a 30 & 3.060 & .712 & 1.197 & 556 & .232 \\
& Mayor o igual a 31 & 2.987 & .722 & & & \\
\hline \multirow{2}{*}{ Evitativo } & Menor o igual a 30 & 3.029 & .744 & 0.331 & 556 & .741 \\
& Mayor o igual a 31 & 3.006 & .845 & & & \\
\hline \multirow{2}{*}{ Dominante } & Menor o igual a 30 & 3.051 & .706 & 1.336 & 556 & .182 \\
& Mayor o igual a 31 & 2.970 & .711 & & & \\
\hline
\end{tabular}


el conflicto, más allá de las consecuencias que esto traiga. Sirivum (2001) sugirió que el estilo integrador tiene dos elementos distintivos: la confrontación y la resolución del problema. La primera involucra comunicación abierta y directa hasta llegar a una vía que permita solucionar el problema. El uso de este estilo puede traer como resultado soluciones creativas a los problemas.

Según Rahim $(1983,2000)$ el estilo integrador suele ser, de forma general, la manera más apropiada de manejar los conflictos interpersonales en el ambiente organizacional, debido a que estas personas tienden a examinar las diversas partes involucradas, antes de tomar decisiones.

Los hallazgos obtenidos en la presente investigación, coinciden parcialmente con lo señalando por Granell, Garaway \& Malpica (1997), quienes reportan que el conflicto interpersonal en las organizaciones tiende a ser visto por los gerentes venezolanos como algo que sería mejor eliminar, evitar y esconder, ya que puede significar herir los sentimientos del otro, exponerlo a la crítica y correr el riesgo de perder la amistad, lo cual es muy valorado en el medio laboral. Sin embargo, sugieren que aunque menos frecuentemente, también se encuentra gerentes que se inclinan a ver el conflicto y las diferencias como una oportunidad para enriquecerse, "ganar a partir de las diferencias"; es decir, esta segunda característica reportada por Granell et al. se vincula estrechamente con el estilo integrador utilizado por los gerentes del Área Metropolitana de Caracas.

El uso predominante del estilo integrador y del estilo comprometido como segundo estilo más frecuente coincide con lo reportado en investigaciones de otros países y culturas. En Norteamérica, investigadores como Rahim (1983); Ting-Toomey, Gao, Trubisky, Yang, Kim, Lin \& Nishida (1991); Elsayed-Ekhoulv \& Buda (1996); y Hammond (1999) coinciden en señalar que el estilo integrador resultó ser el más frecuentemente utilizado por los gerentes norteamericanos. Sin embargo, con relación al segundo estilo más utilizado, no existe acuerdo; Rahim (1983) y Hammond (1999) encontraron que fue el estilo complaciente, mientras que Ting-Toomey (1988) encontró que el segundo estilo fue el comprometido.

De la propuesta teórica de Rahim (1983) se puede inferir que el manejo integrador del conflicto constituye un perfil de competencia ideal para situaciones en las cuales se necesita afrontar asuntos organizacionales complejos, sintetizar múltiples ideas y plasmarlas en soluciones, especialmente cuando el compromiso de todas las partes resulta necesario para implementar la solución o cuando los recursos para la solución están distribuidos en diversas partes, es decir, una sola parte no puede solucionar el problema. Sin embargo, las personas de estilo integrador suelen ser menos apropiadas cuando las tareas o los problemas son simples o cuando a las otras partes involucradas en el conflicto no les interesa la resolución.

En relación a las características demográficas (género y edad), se obtuvo que el género no influye sobre los estilos de manejo de conflicto, estos resultados coinciden parcialmente con los reportados por Rahim (1983) y Ting-Toomey (1988), quienes señalan que el estilo integrador resultó ser el más utilizado por ambos géneros. A excepción de Rahim (1983), que reporta que las mujeres se comportan de manera más integradora, evitativas y comprometidas que los hombres, el resto de las investigaciones no encuentran diferencias estadísticamente significativas en el uso del estilo entre los géneros, es decir, no encuentran influencia del género sobre el estilo de manejo de conflicto.

En relación a la edad de los gerentes, se encontró que ésta no influye sobre el estilo de manejo de conflicto integrador. Asimismo, al evaluar el estilo comprometido no se encontró diferencia significativa según la edad del gerente. Estos resultados coinciden con los reportados por Rahim (1983), quien no encuentra diferencias estadisticamente significativas en la preferencia del estilo según la edad y tampoco encuentra relación entre la intensidad de uso del estilo y la edad de los participantes.

\section{Conclusiones}

Los resultados obtenidos permitieron identificar que el principal estilo de manejo de conflicto utilizado por gerentes del Área Metropolitana de Caracas fue el integrador, el cual tiene como características que la persona intenta relacionar la preocupación concerniente a sí misma como a las otras partes e involucra la colaboración activa entre las diversas posiciones a través del intercambio de información y de analizar las diferencias buscando alcanzar una solución que satisfaga las preocupaciones de ambas partes (Rahim, 2000). Este estilo tiene dos elementos distintivos: la confrontación, lo que involucra una comunicación abierta, y la resolución del problema. Como resultado, dicho estilo puede generar soluciones creativas a los problemas. 
Adicionalmente, se identificó que el segundo estilo de manejo de conflicto más frecuentemente utilizado fue el comprometido, el cual tiene como característica que la persona busca asegurar la obtención de los objetivos organizacionales llegando a decisiones mutuamente aceptables, buscando un punto de equilibrio entre las partes.

Los resultados muestran que el género femenino tiende a comportarse de forma más integradora y comprometido que el género masculino. Con relación a la edad, no se encontró influencia sobre los estilos de manejo de conflicto en los gerentes.

Entre las principales contribuciones del estudio y cumpliendo con los aspectos que justificaron la presente investigación, se señala, en primer lugar, que se logró obtener información específica sobre las tendencias de uso de los estilos de manejo de conflicto interpersonales utilizados por gerentes del Área Me- tropolitana de Caracas. En segundo lugar, se logró determinar la influencia de algunas características demográficas sobre los estilos de manejo de conflicto. Adicionalmente se logró verificar el valor teórico del enfoque sobre estilos de manejo de conflicto desarrollado por Rahim (2000) y su validez en el contexto venezolano.

Los resultados obtenidos en esta investigación constituyen una aproximación inicial acerca de las relaciones existentes entre los estilos de manejo de conflicto y algunas características demográficas en gerentes venezolanos.

Como principal limitación se puede señalar que la muestra de este estudio se circunscribió al Área Metropolitana de Caracas, Venezuela, y a participantes con formación universitaria, lo que implica una limitante para generalizar los hallazgos a toda la población venezolana.

\section{Referencias}

Blake, R.R. \& Mouton, J.S. (1964). Managerial grid. Houston, Texas: Gulf.

ElSayed-Ekhouly, S.M. y Buda, R. (1996). Organizational conflict: A comparative analysis of conflict styles across cultures. International Journal of Conflict Management, $7,71-81$.

Granell, E., Garaway, D. \& Malpica, C. (1997). Exito gerencial y cultura: Retos y oportunidades en Venezuela. Caracas: Instituto de Estudios Superiores Administrativos (IESA).

Hammond, L.J. (1999). Conflict management style preferences of males and females in the role of managers and students. Fort Lauderdale, FL: Nova Southeastern University (disertación doctoral).

Kreitner, R. \& Kinicki, A. (1996). Comportamiento en las organizaciones. Barcelona: Irwin.

Rahim, M.A. (1983). Rahim Organizational Conflict Inventory-II. Palo Alto, CA: Consulting Psychologists Press.

Rahim, M.A. (2000). Do justice perceptions influence styles of handling conflict with supervisors?: what justice perceptions, precisely? International Journal of Conflict Management, 11(1), 9-23.

Rahim, M.A. \& Afza, M. (1993). Leader power, commitment, satisfaction, compliance, and propensity to leave a job among U.S. accountants. Journal of Social Psychology, 133(5), 611-625.
Ramírez, J. \& Álvarez, G (en prensa). Inventario de estilos de manejo de conflictos: Análisis psicométrico. Revista Analogias del Comportamiento.

Robbins, S. (1998). Comportamiento organizacional. México: Prentice Hall.

Sirivum, U. (2001). An Investigation of the primary and secondary conflict management style preferences of men and women in the role of local managers, international managers, and college students in Thailand. Fort Lauderdale, FL: Nova Southeastern University (disertación doctoral).

Thomas, K.W. (1976). Conflict and conflict management. En M.D. Dunnette (Ed.), Handbook of industrial and onganizational psychology (pp. 889-935). Chicago: Rand McNally.

Ting-Toomey, S. (1988). Intercultural conflict styles: A facenegociation theory. En Y. Kim \& W. Gudykunst (Eds.), Theories in intercultural communications (pp. 213-235). Newbury Park, California: Sage.

Ting-Toomey, S., Gao, G., Trubisky, P., Yang, Z., Kim, H.S., Lin, S.L. \& Nishida, T. (1991). Culture, face maintenance and conflict styles of hadling interpersonal conflict: A stu$\mathrm{dy}$ in five cultures. International Journal of Conflict $\mathrm{Ma}$ nagement, 2, 275-296.

Van de Vliert, E. \& Kabanoff, B. (1990). Toward theory-based measures of conflict management. Academy of Management Journal, 33, 199-209. 